\title{
La prueba CTX como evaluador de riesgo en el diagnóstico y tratamiento de osteonecrosis de los maxilares inducida por el uso de bifosfonatos
}

\section{Role of the CTX Test for Evaluation of the Risk in Diagnostic and Treatment of the Osteonecrosis of the Jaws Induced by the use of Bisphosphonates}

Patricia Lorz Ulloa DDS1; Rodolfo Varela Guillén DDS²

\begin{abstract}
1. Universidad de Costa Rica, Especialista en Patología y Cirugía Oral Pontificia Universidad Javeriana, docente Sección de Cirugía Oral y Maxilofacial de la Facultad de Odontología de la Universidad de Costa Rica, Costa Rica 2. Universidad de Costa Rica, Práctica privada en Clínica Dental Santa Bárbara, Heredia.

Pasante Pasantía de Cirugía Oral Universidad de Costa Rica, Costa Rica.

Autor para correspondencia: Dra. Patricia Lorz Ulloa - Iorz@ucr.ac.cr
\end{abstract}

Recibido: 13-III-2014

Aceptado: 08-IV-2015

Publicado Online First: 16-XI-2015

DOI: http://dx.doi.org/10.15517/ijds.v0i0.22044

\section{RESUMEN}

Se realizó una revisión bibliográfica con el fin de conocer la efectividad de la prueba del telopéptido C terminal de reticulación (CTX), la cual ha sido sugerida para predecir el grado de riesgo a padecer osteonecrosis de los maxilares en pacientes que consumen o consumieron bifosfonatos orales. La osteonecrosis de lo maxilares (OMIB) es una condición ósea que provoca necrosis avascular del hueso alveolar y que se da en pacientes que están bajo tratamiento con bifosfonatos. Se ha sugerido que un valor de CTX por debajo de los $100 \mathrm{pg} / \mathrm{ml}$ representa un riesgo alto de padecer OMIB, mientras que entre los 100 y los $150 \mathrm{pg} / \mathrm{ml}$ un riesgo moderado y por encima de los $150 \mathrm{pg} / \mathrm{ml}$ un riesgo mínimo. La prueba CTX ha sido debatida, y no ha sido posible enmarcarla como prueba contundente, ya que diversos estudios han encontrado resultados muy variables, en pacientes que con CTX muy bajos no han desarrollado OMIB y otros que si lo han hecho con valores de CTX muy altos, supuestamente seguros. Conclusión: La prueba CTX es una prueba útil para informar al odontólogo acerca del grado de actividad en el recambio óseo del paciente, y puede ser útil para aconsejar al paciente. Niveles bajos de CTX podrían servir para aplazar un procedimiento quirúrgico que no se considere urgente. Se recomienda aumentar a $200 \mathrm{pg} / \mathrm{ml}$ la línea de riesgo quirúrgico en relación a los $150 \mathrm{pg} / \mathrm{ml}$ recomendados por Marx.

\section{PALABRAS CLAVES}

Prueba CTX, Bifosfonatos, Osteonecrosis de los maxilares, Prevención, Riesgo quirúrgico.

LORZ P. \& VARELA R., 2015: La prueba CTX como evaluador de riesgo en el diagnóstico y tratamiento de osteonecrosis de los maxilares inducida por el uso de bifosfonatos.-ODOVTOS-Int. J. Dental Sc., 17-1 (January-April): 41-51. 


\section{ABSTRACT}

A literature review was conducted to determine the effectiveness of the C-terminal crosslinking telopeptide (CTX) serologic test, which has been suggested to predict the risk of developing osteonecrosis of the jaw in patients taking oral bisphosphonates. Osteonecrosis of the jaw (ONJ) is a condition that causes avascular necrosis of the alveolar bone, which occurs in patients treated with bisphosphonates. It has been suggested that a CTX value below $100 \mathrm{pg} / \mathrm{ml}$ represents a high risk for ONJ , while between 100 and $150 \mathrm{pg} / \mathrm{ml}$ a moderate risk and above 150pg/ml minimal risk. The CTX test has been controversial and it has not been possible to frame it as strong evidence, since several studies have found variable results. Patients with very low CTX levels have not developed ONJ, and others with very high CTX levels, supposedly safe, have done. CTX test is a useful test to inform the dentist about the activity level on patient's bone turnover, and may be useful to advise the patient. Low CTX levels could serve to delay a surgical procedure that is not deemed urgent.

\section{KEYWORDS}

CTX test, Bisphosphonates, Osteonecrosis of the jaws, Prevention, Surgical risk.

\section{INTRODUCCIÓN}

Desde que Robert E. Marx et al. en el año 2007 publica un artículo acerca de la efectividad de la prueba del telopéptido $C$ terminal, como predictor para determinar el riesgo para un paciente de desarrollar Osteonecrosis de Maxilares Inducida por Bifosfonatos (OMIB), relacionado con procedimientos dentales invasivos que involucraran hueso, se ha levantado una controversia ya que se ha debatido su validez y efectividad con respecto a los resultados finales $(1,2,3)$.

El objetivo del artículo es presentar una revisión bibliográfica tomando en cuenta los estudios relacionados y discutir su efectividad para conocer el riesgo de padecer osteonecrosis de los maxilares después de realizar procedimientos quirúrgicos en cavidad oral.

El uso de bifosfonatos para el tratamiento de diversas condiciones óseas se ha incrementado alrededor del mundo. En Estados Unidos por ejemplo, alcanzó las 225 millones de prescripciones en el 2007 $(4,5,6)(3,4,5)$. En 1980 se descubrió la eficacia de los bifosfonatos para la prevención de fracturas vertebrales en mujeres postmenopáusicas. Desde ese momento, han sido utilizados para el tratamiento de osteoporosis relacionadas con glucocorticoides, enfermedades metastásicas en hueso, mieloma múltiple, hipercalcemia, enfermedad de Paget, y como radionucleótido (technetium 99 difosfonato) en cintigrafía ósea. $(4,7,8)$ Actualmente existe una gran variedad de bifosfonatos, éstos se pueden dividir en nitrogenados, no nitrogenados, orales e intravenosos, cada uno con mayor o menor grado de riesgo para poder causar osteonecrosis de los maxilares.

\section{BÚSQUEDA DE LITERATURA}

Se utilizaron los motores de búsqueda EBSCOhost, Adonis, Medline, Clinicalkey y Elsevier. Los criterios de inclusión utilizados fueron: artículos de publicación reciente, en revistas científicas en el área de la odontología y bioquímica que estuvieran enfocados en el tema de la revisión de literatura. Las palabras clave que se introdujeron para encontrar la información fueron: osteonecrosis de maxilares, bifosfonatos, 
prueba del telopéptido c terminal de reticulación, CTX, riesgo quirúrgico, prevención.

\section{OSTEONECROSIS DE LOS MAXILARES INDUCIDA POR BIFOSFONATOS (OMIB)}

La OMIB es una condición ósea que provoca necrosis avascular del hueso alveolar y se presenta en pacientes que están bajo tratamiento con bifosfonatos ya sean orales o intravenosos. Esta patología, se puede presentar a partir de un procedimiento dental invasivo, trauma, o de forma espontánea y afectará únicamente a los maxilares. (8) La OMIB es 70\% más común en mujeres, a quienes se les prescribe con más frecuencia este tipo de medicamento (9).

La Asociación Americana de Cirujanos Orales y Maxilofaciales (AAOMS), define a la OMIB como un área de exposición ósea en la región maxilofacial que ha persistido por más de 8 semanas en un paciente que actualmente o previamente ha estado en tratamiento con bifosfonatos y sin historia de radioterapia en los maxilares (10).

Son factores de riesgo para la adquisición de OMIB la potencia del bifosfonato, vía de administración, duración del tratamiento, cirugía dentoalveolar, incremento de la edad, comorbilidades médicas, factores genéticos y debilidad $(4,9,11,12)$. Entre los detonantes orales, el $75 \%$ fue por extracciones dentales, seguido de enfermedad periodontal y prótesis desajustadas (9).

Los pacientes con historia de bifosfonatos intravenosos tienen un riesgo a desarrollar una osteonecrosis de $0.88 \%$ a $1.15 \%$. Este valor se incrementa después de una extracción dental de 6.7 $\%$ a $9.1 \%$. En pacientes expuestos a bifosfonatos orales el porcentaje es de $0.01 \%$ a $0.04 \%$ con un incremento de $0.09 \%$ a $0.34 \%$ después de una extracción dental (11). Además, se estima que para los bifosfonatos orales el riesgo de provocar
OMIB entre el primer y tercer año es bajo y para los intravenosos serán los primeros meses (9).

\section{TELOPÉPTIDO C TERMINAL DE RETICULACIÓN (CTX)}

En el 2007, Marx y colaboradores sugieren una prueba diagnóstica para conocer el riesgo que corren los pacientes bajo tratamiento con bifosfonatos orales y/o corticosteroides por más de 3 años de tomar el medicamento de padecer OMIB. Este análisis se llama telopéptido $\mathrm{C}$ terminal de reticulación o CTX, es determinado en plasma.

Esta prueba fue sugerida por Rosen y colaboradores en el año 2000, como un método para monitorear a los pacientes que recibían terapia antiresortiva, a la hora de que se les administraban bifosfonatos, además para el control de la terapia hormonal antiresortiva en mujeres post menopáusicas pero Marx lo sugiere para medir el riesgo de padecer $\operatorname{OMIB}(8,13,14)$.

La medición sérica proporciona una ventaja con respecto al ensayo en orina porque evita el efecto aditivo de la variabilidad biológica de la excreción de creatinina urinaria. $(9,15,16)$ Mediante un índice biológico ( $\mathrm{Pg} / \mathrm{ml}$ picogramos por mililitro) mediría la remodelación ósea y la resorción como un parámetro de la actividad osteoclástica. Los bifosfonatos realizan una acción farmacológica mediante la disminución de la actividad osteoclástica, el $90 \%$ de la matriz orgánica del hueso es de colágeno tipo 1 (7). Durante la resorción ósea, los osteoclastos crean lagunas de resorción ósea con microambientes de pH bajos, donde se disuelve la matriz inorgánica exponiendo la matriz orgánica. A medida que la resorción ósea avanza, las enzimas de los osteoclastos digieren la matriz ósea orgánica liberando productos de colágeno tipo I, Ios cuales incluyen fragmentos de telopéptido C-terminales del colágeno tipo I llamados CTX y anillos estructurales llamados crosslink de piridinolina. 
Durante el metabolismo normal de los huesos, el colágeno maduro tipo 1 se degrada y pequeños fragmentos pasan a la sangre y se excretan vía renal. Como control, se ha sugerido la medición del CTX antes de comenzar el tratamiento con bifosfonatos, ya que el nivel se va a reducir en un $60 \%$ a las 6 semanas, cuando se usa una dosificación convencional. (1) En tanto exista un recambio óseo más veloz, el nivel de CTX será más alto como en el caso de la enfermedad de Paget, pero si la tasa de resorción ósea es baja entonces el CTX será bajo también. Se ha establecido que los valores de CTX por debajo de los 100 pg/ml representaban un riesgo alto de padecer OMIB, mientras que entre los 100 y los $150 \mathrm{pg} / \mathrm{ml}$ un riesgo moderado y por encima de los $150 \mathrm{pg} / \mathrm{ml}$ un riesgo mínimo o ningún riesgo. El CTX, ha sido utilizado en medicina para medir el recambio óseo en enfermedades como osteoporosis, metástasis óseas como respuesta a los bifosfonatos y se emplea como un parámetro de efectividad de la terapia de bifosfonatos orales.

Sin embargo, esta prueba ha sido muy cuestionada ya que el estudio de donde se origina no cuenta con suficiente respaldo científico. (17) También ha sido criticado ya que la gran mayoría de los pacientes que toman bifosfonatos orales no padecen de OMIB, a pesar de estar en los niveles de peligro. Del estudio, se puede criticar el hecho de no haberse establecido un grupo control de pacientes que también estuvieran tomando bifosfonatos orales pero que estuvieran sin OMIB. Por estas razones,se ha cuestionado la prueba del CTX como medio predictorio de OMIB. De la prueba, se ha debatido que el CTX es una prueba que mide el recambio óseo corporal, por lo que es difícil que pudiera determinar un parámetro específico para los maxilares, no se conoce con exactitud el porcentaje, pero es claro que es pequeño en relación con el recambio total corporal y que la velocidad de recambio óseo alveolar es diez veces más rápida que en huesos largos (9).

\section{ANTECEDENTES}

0'Connell et al. (2012) En los resultados ninguno de los pacientes desarrolló OMIB. Concluye este estudio que el CTX no fue predictivo para el desarrollo de OMIB luego de la cirugía oral, y no fue concluyente apoyarse en la prueba de CTX para iniciar o detener un tratamiento (4).

Kunchur et al. (9) concluye que los resultados no demuestran una gran efectividad de la prueba CTX, pero si sirve para calcular el tiempo que un paciente debe de suspender el medicamento para llegar a un valor de CTX seguro. Al suspender la toma del fármaco, los rubros de CTX aumentarán un 26,4 pg/ml al mes según Marx (1). Consideró que de los 61 pacientes, 28\% tenían un CTX por encima de los $300 \mathrm{pg} / \mathrm{ml}$, básicamente por dos situaciones: que no se tomaban el medicamento y la otra por las diferentes respuestas metabólicas. Recomienda que los estudios deben realizarse con muestras más grandes, con un tiempo mayor de observación y a pesar de que las casas farmacéuticas reportan una bajísima incidencia de OMIB orales, si se deben tomar las previsiones de tratamiento oral quirúrgico antes de comenzar un tratamiento de este tipo, especialmente si son intravenosos, y que la prueba de CTX no es predictiva pero puede ayudar para clasificar a los pacientes dentro de una línea de riesgo que está por lo niveles menores de $200 \mathrm{pg} / \mathrm{ml}$ y no de 150 $\mathrm{pg} / \mathrm{ml}$ citada por Marx et al. (1).

Bagan et al. (18) realizaron un estudio retrospectivo en 15 pacientes, en pacientes bajo tratamiento con bifosfonatos intravenosos, los cuales es sabido tienen una mayor potencia que los orales y aunque el pamidronato es 
comparable en potencia con el alendronato (1), la recomendación es hacer estudios con mayor número de pacientes que estén bajo tratamiento exclusivo de bisfofonatos intravenosos.

Kwon et al. (19), realizaron un estudio con 18 pacientes diagnosticados con osteonecrosis de maxilares causada por bifosfonatos orales, estudiaron los valores de CTX de los pacientes, lo cuales oscilaron entre 10 y $262 \mathrm{pg} / \mathrm{ml}$, con una media de 112 +_ 76.1 , el promedio de tiempo de ingesta del fármaco fue de 3.9 años, con dosis semanales de $70 \mathrm{mg}$ de alendronato en 17 de los 18 pacientes y uno en dosis semanal de risedronato de $35 \mathrm{mg}$. Las edades oscilaban entre los $61 \mathrm{y}$ los 86 años, con promedio 74,39. Se tomaron los parámetros de riesgo propuestos por Marx, se clasificaron según número de lesiones, estadio de la lesión, localización de la lesión y se compararon con niveles de CTX de los pacientes. Encontraron resultados estadísticamente significativos entre la severidad de la lesión y la evaluación de riesgo utilizando CTX.

Lazarovici (20) indica que por la amplia variabilidad de los resultados no se puede sugerir que los niveles de CTX se puedan utilizar como el marcador predictivo final para determinar si un paciente puede 0 no desarrollar OMIB. Sin embargo, se puede decir que cuando el nivel de CTX es menor de $150 \mathrm{pg} / \mathrm{ml}$, el riesgo de desarrollar la patología aumenta significativamente tal como lo había citado Marx (1).

Lee (21), no encontró una relación directa entre los valores del CTX y el potencial para desarrollar OMIB, basado en que los 21 pacientes con valores menores de $100 \mathrm{pg} / \mathrm{ml}$ que se realizaron un procedimiento y no suspendieron el medicamento, ninguno de ellos desarrolló OMIB.

Concluye que el uso de marcadores químicos no tiene validez para evaluar con precisión el nivel de riesgo de desarrollar OMIB y que no hubo correlación entre comorbilidades y la patología.

Passof (5) realiza una revisión bibliográfica para determinar la predictibilidad del CTX en el riesgo de padecer OMIB, encontrando 11 artículos relacionados directamente con el problema. De éstos, 8 documentos no reportaron una relación directa entre el CTX y la efectividad como herramienta para predecir una OMIB. Solo 3 artículos si encontraron una relación directa, incluido entre éstos el de Marx del 2007 (1). Los otros dos del mismo grupo de investigadores.

Avolio et al. (22) realizaron un estudio para determinar si el CTX serológico es útil para indicar el nivel de actividad metabólica del hueso mandibular. Seleccionaron treinta y seis pacientes, de los cuales 10 se encontraban sanas y 26 enfermas, todas eran posmenopáusicas y mayores de 50 años 0 más. Se utilizó la densitometría mineral ósea (DM0) como criterio para la selección de los pacientes con masa ósea normal y anormal. Se analizó la captación de radioisótopos en el cuello femoral (R2) y el cuerpo mandibular (R1), además del CTX serológico. Pudieron comprobar que los pacientes que presentaron una DMO baja, como consecuencia de la posible elevación de la actividad de resorción, presentaron niveles de CTX altos, producto del bajo nivel de la remodelación ósea y este marcador si es útil para medir la actividad metabólica en mandíbula.

\section{CONCLUSIONES}

La prueba CTX no demostró una gran efectividad como predictor definitivo de riesgo de padecer osteneocrosis de los maxilares inducida por bifosfonatos, pero es útil para calcular el tiempo en que se debe suspender el medicamento para alcanzar niveles más altos de recambio óseo y disminuir así la posibilidad de padecer OMIB después de una extracción o cirugía oral. 
Por lo tanto es una prueba útil para informar al odontólogo acerca del grado de actividad en el recambio óseo y así poder aconsejar al paciente.

A pesar de que Marx (1) estableció que al suspender el bifosfonato oral, el valor de CTX aumentaría 26,4 pg/ml al mes, esa aseveración podría discutirse ya que otros investigadores demostraron que el incremento del CTX por mes puede ser variable.
El CTX no es predictivo, pero puede ayudar a clasificar a los pacientes dentro de una línea de "seguridad" la cual debería estar por arriba de los $200 \mathrm{pg} / \mathrm{ml}$.

Los niveles bajos de CTX podrían servir para aplazar un procedimiento quirúrgico que no se considere urgente. Se recomienda aumentar a 200 pg/ml la línea de riesgo quirúrgico en relación a los $150 \mathrm{pg} / \mathrm{ml}$ recomendados por Marx. 


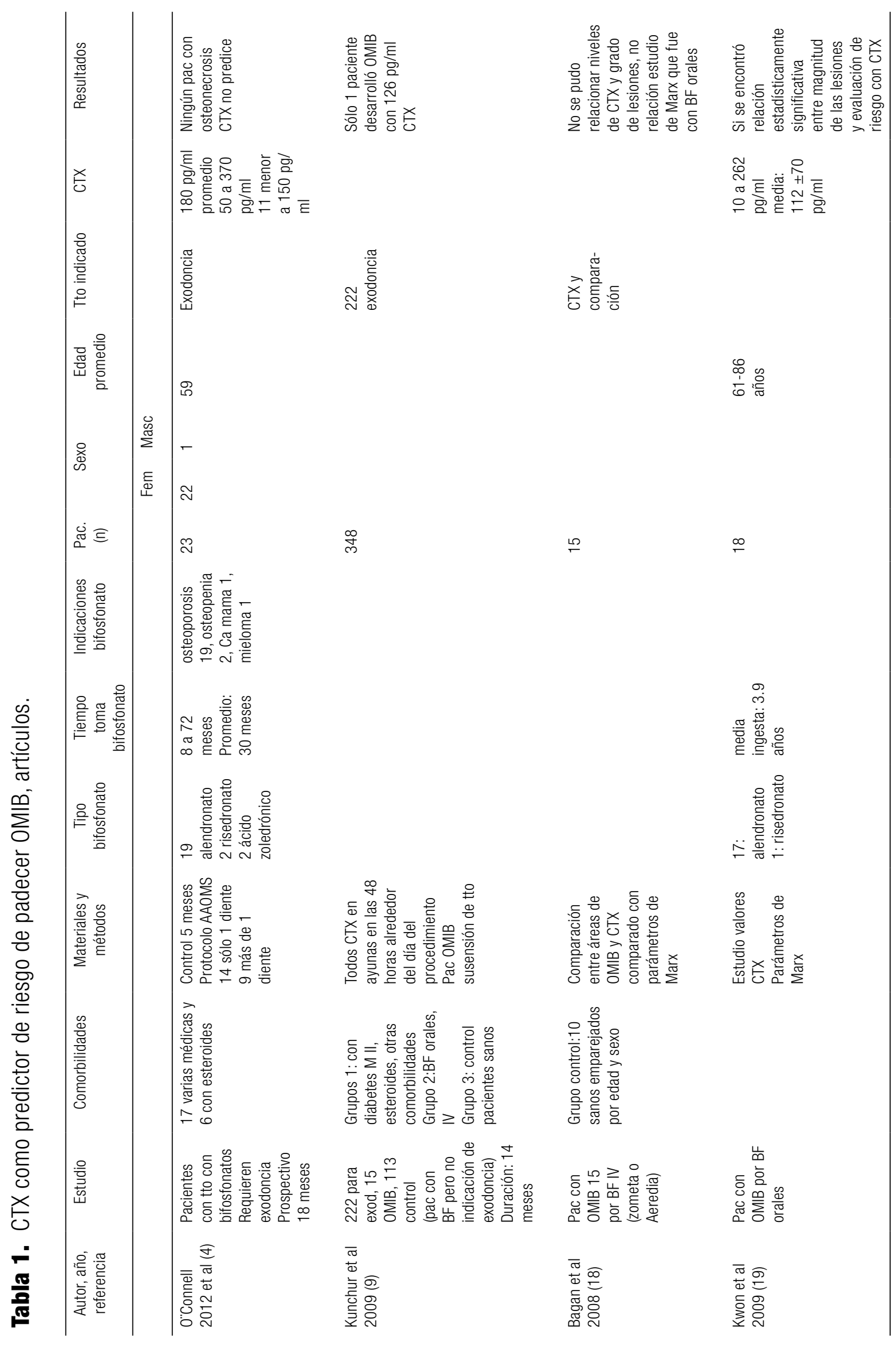




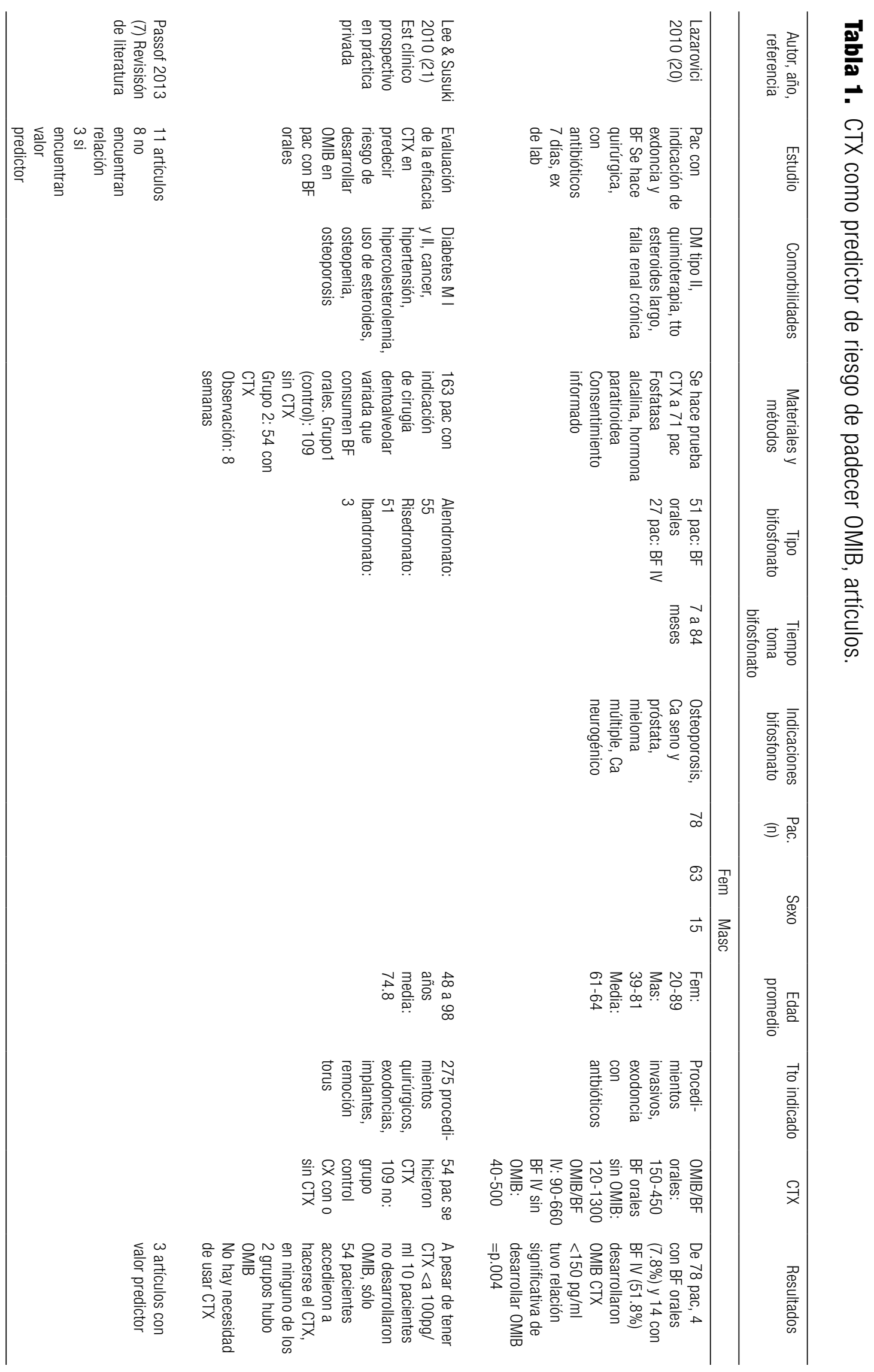




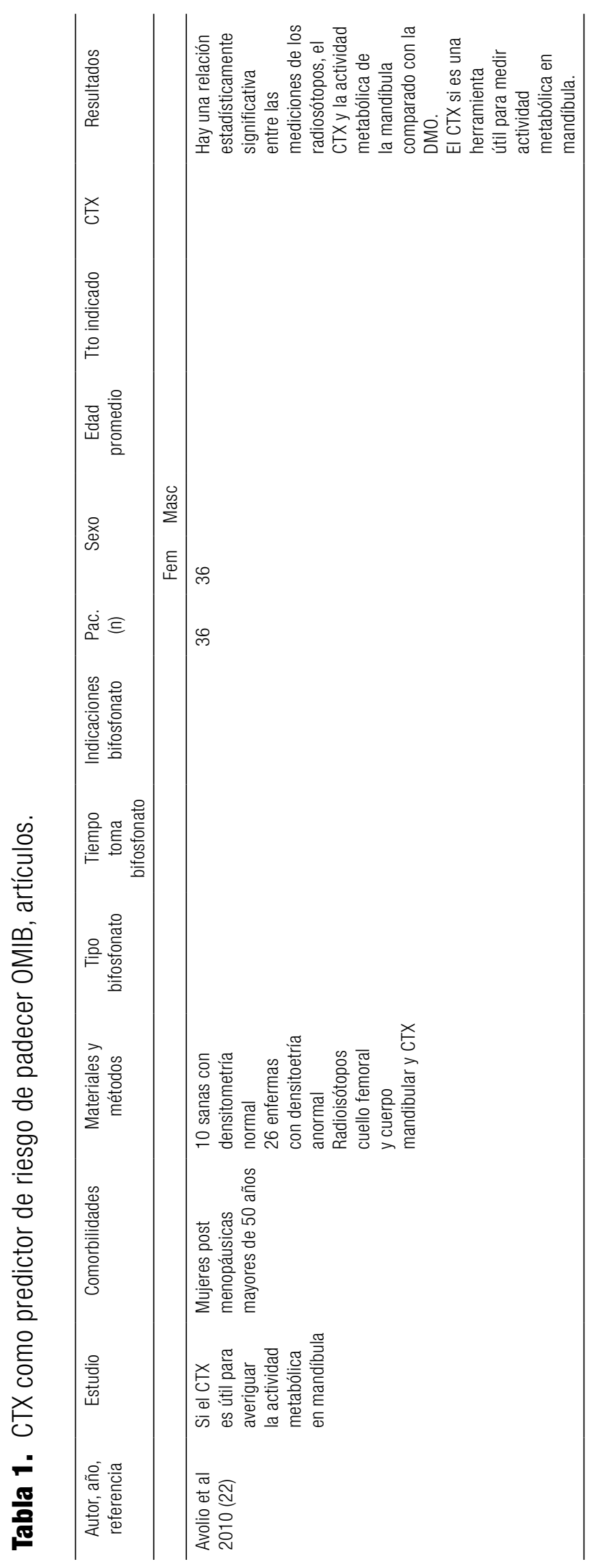




\section{REFERENCIAS}

1. Marx R., Cillo J. Jr., Ulloa J. J. Oral bisphosphonate-induced osteonecrosis: risk factors, prediction of risk using serum CTX testing, prevention, and treatment. Journal of Oral and Maxillofacial Surgery, Dec 2007; 65(12): 2397-2410.

2. Marx R. E., Sawatari Y, Fortin M, Broumand V. Bisphosphonate-induced exposed bone (osteonecrosis/ osteopetrosis) of the jaws: Risk factors, recognition, prevention, and treatment. Journal of Oral and Maxillofacial Surgery, 2005; 63, 1567.

3. Assael LA. Serum CTX to prevent osteonecrosis/orthodontic extraction of third molars: paths toward minimizing surgical risk?. Journal of Oral and Maxillofacial Surgery, (Dec-2007;65(12): 2395-2396.

4. O'Connell J. E., Ikeagwani O, Kearns G. J. A role for C-terminal cross-linking telopeptide (CTX) level to predict the development of bisphosphonate-related osteonecrosis of the jaws (BRONJ) following oral surgery? Irish Journal of Medical Science, Jun-2012; 181(2): 237-242.

5. Pasoff M. C-Terminal Cross-Linking Telopeptide as a Serologic Marker for Bisphosphonate-Related Osteonecrosis of the Jaw: Review of 2 Cases. Journal of the Canadian Dental Association, (2013). 1-11.

6. Sawatari Y., Marx R. E. Bisphosphonates and Bisphosphonate Induced Osteonecrosis. Oral Maxillofacial, Surgical Clinics of North America Journal, 2007; 19: 487-498.

7. Lazarovici T. S., Mesilaty-Gross S., Vered I., Pariente C., Kanety H., Givol N,. Yahalom R., Taicher S., Yarom N. Serologic bone markers for predicting development of osteonecrosis of the jaw in patients receiving bisphosphonates. Journal of Oral and Maxillofacial Surgery, Sep-2010;68(9): 2241-2247.
8. Lee C.Y., Suzuki J.B.CTX biochemical marker of bone metabolism. Is it a reliable predictor of bisphosphonate-associated osteonecrosis of the jaws after surgery? Part I: biological concepts with a review of the literature. Implant Dentistry, Dec-2009;18(6): 492-500.

9. Kunchur R, Need A, Hughes T, Goss A. Clinical investigation of $\mathrm{C}$-terminal cross-linking telopeptide test in prevention and management of bisphosphonate-associated osteonecrosis of the jaws. Journal of Oral and Maxillofacial Surgery, Jun-2009; 67(6): 1167-1173.

10. Ruggiero S. L., Dodson T. B., Assael L.A., Landesberg R, Marx R. E., Mebrotra B. American Association of Oral and Maxillofacial Surgeons position paper on bisphosphonaterelated osteonecrosis of the jaws. Journal of Oral and Maxillofacial Surgery, 2009; 67, 2.

11. Borromeo G. L., C. E. Tsao, I.B. Darby, P.R Ebeling. A review of the clinical implications of bisphosphonates in dentistry. Australian Dental Journal, 2011; 56: 2-9.

12. Mavrokokki T, Cheng A, Stein B, Goss A. Nature and frequency of bisphosphonateassociated osteonecrosis of the jaws in Australia. Journal of Oral and Maxillofacial Surgery, 2007; 65: 415-423.

13. Dodson T. B. CTX and its role in managing patients exposed to oral bisphosphonates. Journal of Oral and Maxillofacial Surgery, Feb-2010; 68(2): 487-488.

14. Khosla S, Burr D., Cauley J., Dempster D.W, Ebeling P, Felsenberg D., Gagel R. Oral bisphosphonate-induced osteonecrosis: Risk factors, prediction of risk using serum CTX testing, prevention, and treatment. American Society for Bone and Mineral Research Task Force on Osteonecrosis of the jaw. Journal of Oral and Maxillofacial Surgery, (2008); 66, 1320.

15. Reynag B., Zeni S. Marcadores bioquímicos del remodelamiento óseo. Utilidad clínica 
Acta Bioquímica Clínica Latinoamericana, Abril-Junio 2009; 43 (2): 177-193.

16. Cons F. Marcadores Bioquímicos de Remodelado Óseo. Revista de Metabolismo Oseo y Mineral, 2003;1(3): 91-98.

17. Schwartz H. C. Serum CTX testing. Journal of Oral and Maxillofacial Surgery, Jun-2008; 66(6): 1319-1320.

18. Bagan J.V., Jiménez Y., Gómez D., Sirera R., Poveda R., Scully C. Collagen telopeptide (serum CTX) and its relationship with the size and number of lesions in osteonecrosis of the jaws in cancer patients on intravenous bisphosphonates. Oral Oncology, Nov 2008; 44(11).

19. Kwon Y., Kim D., Ohe J., Yoo J., Walter C. Correlation between serum C-terminal cross-linking telopeptide of type I collagen and staging of oral bisphosphonate-related osteonecrosis of the jaws. Journal of Oral and Maxillofacial Surgery, Dec- 2009; 67(12): 2644-2648.

20. Lazarovici T. S., Yahalom R., Taicher S., Elad S., Hardan I., Yarom N. Bisphosphonaterelated osteonecrosis of the jaws: A singlecenter study of 101 patients. Journal of Oral and Maxillofacial Surgery, 2009;67: 850-855.

21. Lee C.Y. CTX biochemical marker of bone metabolism. Is it a reliable predictor of bisphosphonate-associated osteonecrosis of the jaws after surgery? Part II: a prospective clinical study. Implant Dentistry, Feb2010;19(1): 29-38.

22. Avolio G.,Brandão C., Marcucci M.,Alonso G. Use of the plasma CTX for assessing the bone activity of the mandible among osteopenic and osteoporotic patients. Brazillian Oral Research, 2010; 24(2): 250-255. 